\title{
Propagation Loss Reduction Between On-Body Antennas by Using a Conductive Strip Line
}

\author{
Tran Thi Lan (1), Member, IEEE, and Hiroyuki Arai ${ }^{\circledR}$, Fellow, IEEE
}

\begin{abstract}
This letter proposes a very simple solution to reduce the propagation loss between two antennas/sensors on body at 2.45 GHz by utilizing a flexible conductive strip line (CSL). The performance of the CSL is comparable to that of the free-access transmission line in the work by Tran et al. in some certain cases. The transmission improvement by the CSL is verified by measurement results on a perfect electrical conductor plane, on a phantom, and on a real body more than $20 \mathrm{~dB}$ for non-line-of-sight scenarios. Dimension parameters of the CSL are optimized so that the highest improvement by the CSL can be achieved and it is easy to be integrated into clothes. Moreover, the specific absorption rate is satisfied when the CSL is deployed near body.
\end{abstract}

Index Terms-Electromagnetic coupling, on-body communication, paper substrate, silver ink, smart suit, transmission line.

\section{INTRODUCTION}

$\mathbf{O}$ N-BODY communication has attracted researchers by its essential applications for healthcare, entertainment, or military. Transmission characteristics between antennas/sensors in on-body communication are negatively affected by human body [1], [2]. Moreover, the propagation loss reduction between the sensors is strongly requested for low power operation. There are some solutions for this problem, such as using flexible cables, wireless connections, reception diversity [17], or widening the ground plane size of the antennas [18]. But, these ways make the system complicated and less flexible. A more favorite way is by utilizing the subsidiary waveguides that are integrated into clothes as smart suits [3]-[8]. On-body antennas communicate with each other by coupling with these smart suits. In other words, smart suits work as low-loss channels for the antennas on body. Thus, high transmission gain is achieved. Besides, high security transmission can be obtained because wearers use personal smart suits. A comparison between these methods is given in Table I. In this letter, a conductive strip line (CSL) is proposed, as shown in Fig. 1, to replace these waveguides. The CSL has a high flexibility by utilizing a paper substrate and a silver-ink conductive layer and an extremely simple fabrication by printing technology. It reduces the loss between on-body antennas more than $20 \mathrm{~dB}$. This is comparable with the free-access transmission line in [7] and [8].

\section{COMPARISON}

To emulate the transmission line in [7] and [8], the CSL has a dimension equivalent to that of the transmission line. It is $19 \mathrm{~mm}$

Manuscript received September 10, 2018; accepted October 14, 2018. Date of publication October 24, 2018; date of current version November 29, 2018. (Corresponding author: Tran Thi Lan.)

The authors are with the Electrical and Computer Engineering Department, Yokohama National University, Yokohama 240-8501, Japan (e-mail: tranlanhp 83@gmail.com; arai-hiroyuki-vk@ynu.ac.jp).

Digital Object Identifier 10.1109/LAWP.2018.2877772
TABLE I

COMPARISON BETWEEN METHODS FOR ON-BODY COMMUNICATION

\begin{tabular}{|l|l|l|}
\hline \multicolumn{1}{|c|}{ Methods } & \multicolumn{1}{|c|}{ Advantages } & \multicolumn{1}{c|}{ Disadvantages } \\
\hline $\begin{array}{l}\text { Reception } \\
\text { diversity [17] }\end{array}$ & $\begin{array}{l}\text { Transmission gain is 7.9 } \div \\
12.7 \mathrm{~dB} \text {. }\end{array}$ & $\begin{array}{l}\text { Less useful in NLOS } \\
\text { case. }\end{array}$ \\
\hline Flexible cables & $\begin{array}{l}\text { Connections between } \\
\text { sensors are reliable. }\end{array}$ & $\begin{array}{l}\text { Complicated when the } \\
\text { number of sensors/ } \\
\text { antennas is large; Not } \\
\text { flexible. }\end{array}$ \\
\hline $\begin{array}{l}\text { Increase the } \\
\text { ground plane } \\
\text { size of } \\
\text { antennas [18] }\end{array}$ & $\begin{array}{l}\text { Performance of the } \\
\text { antennas/ sensors affects } \\
\text { little by body. }\end{array}$ & Less flexible. \\
\hline $\begin{array}{l}\text { Subsidiary } \\
\text { waveguides } \\
\text { [3]-[8] }\end{array}$ & $\begin{array}{l}\text { High improvement more } \\
\text { than 20 dB; Flexible; High } \\
\text { security; Not sensitive with } \\
\text { body postures. }\end{array}$ & $\begin{array}{l}\text { Users may need to wear } \\
\text { special clothes. }\end{array}$ \\
\hline
\end{tabular}

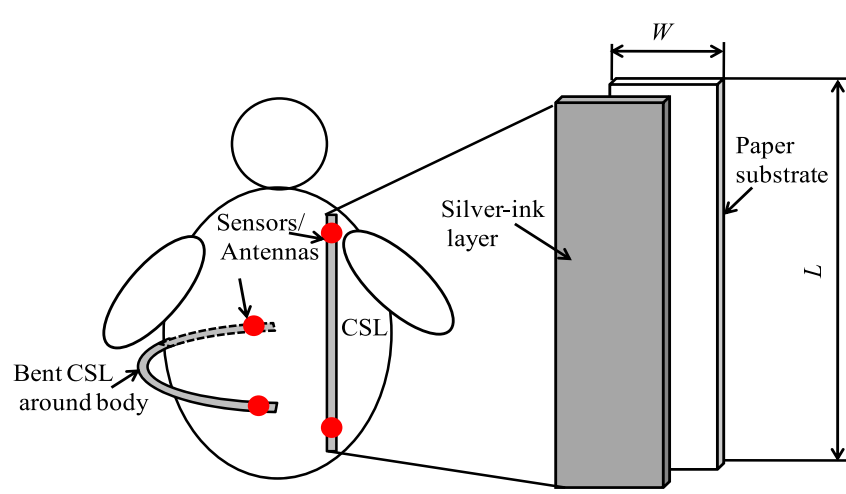

Fig. 1. Deployment of the CSL on a body.

in width and $430 \mathrm{~mm}$ in length. Moreover, the material used to fabricate the CSL is also the same as that of the transmission line with the paper substrate and the silver ink. The thickness of the paper substrate is $0.18 \mathrm{~mm}$ with a tangent loss of $\tau=0.15$ and a dielectric constant of $\varepsilon_{\mathrm{r}}=2.8$, whereas the silver ink is $9 \mu \mathrm{m}$ in thickness with a conductivity of $\sigma=1.5 \times 10^{7} \mathrm{~S} / \mathrm{m}$.

The performance of the CSL at $2.45 \mathrm{GHz}$ is evaluated by calculating the transmission characteristics between the two square patch antennas $\left(50 \times 50 \times 0.8 \mathrm{~m}^{3}\right)[8],[16]$ on the perfect electrical conductor $(\mathrm{PEC})$ plane $\left(750 \times 400 \times 1 \mathrm{~mm}^{3}\right)$ considered as a body [8]. The EMPro software [9] is used for simulation. Three deployments of the CSL are discussed, depending on positions of the patch antennas, as shown in Fig. 2.

To make an equivalent comparison with the transmission line, the models for simulation are also similar to those used in [7] and [8]. The distance between the antennas and the CSL is $1 \mathrm{~mm}$, whereas the distance between the PEC plane and the CSL is $7 \mathrm{~mm}$, which is the minimum distance that the effect of body 


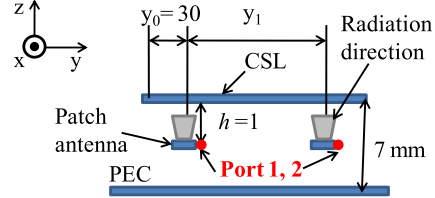

(a)

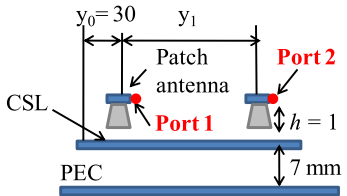

(b)

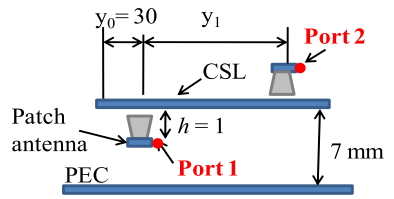

(c)

Fig. 2. Models used to consider transmission characteristics between two patch antennas with CSL. (a) Antennas below the CSL. (b) Antennas above the CSL. (c) Antennas at different sides of the CSL.

on the transmission line is neglected. The patch antenna with port 2 is moved along the CSL in $30 \mathrm{~mm}$ intervals, whereas the antenna with port 1 is fixed. The coupling between the antennas in $\mathrm{S}_{21}$ is calculated at each distance of $y_{1}$, which is the distance between the two antennas. Besides, the coupling between the antennas without the CSL is calculated to get the transmission improvement by the CSL. Here, the transmission improvement is the difference in $\mathrm{S}_{21}$ in two cases: with (w/CSL) and without the CSL (w/o CSL). Simulation results are shown in Fig. 3. The CSL has a really good performance when the antennas are placed below it as shown in Fig. 3(a), the loss reduction is more than $20 \mathrm{~dB}$ for $y_{1}$ large. In the case as shown in Fig. 3(b), the improvement by the CSL is not significant, whereas it is a little in the case as shown in Fig. 3(c). Thus, the CSL is effective in the certain cases. In next sections, we only consider the performance of the CSL in the case as shown in Fig. 3(a).

To get how the CSL works, current distribution of the CSL is shown in Fig. 4, where the CSL works as a microstrip line fed by the patch antennas.

\section{OPTIMAL DIMENSION PARAMETERS}

This section presents a process to get the optimal dimension for the CSL. We did simulations to find the optimal height of $h$ from the antenna to the CSL, the width of $W$, and the length of $L$, respectively. The targets when optimizing the dimension of the CSL are that the loss reduction is more than $20 \mathrm{~dB}$ and the smaller width is the better to get the flexibility. The model is utilized for the simulations as shown in Fig. 5. The distance between the CSL and the PEC plane is $1.2 \mathrm{~mm}$, and the distance from the center of the antenna with port 1 to one end of the CSL is $y_{0}=30 \mathrm{~mm}$ to ensure that this antenna is in the coverage of the CSL. The simulations are done on both the PEC plane and a simplified liquid phantom model [10] for a good comparison. While the PEC plane is for easy fundamental measurements, results on the phantom model are more similar to results on real bodies. The size of the phantom model is $40 \times 75 \times 5 \mathrm{~cm}^{3}$ with the dielectric constant of 39.2 and the conductivity of $1.8 \mathrm{~S} / \mathrm{m}$ [10].

\section{A. Optimal Height of $h$}

The CSL with the equivalent dimension as the transmission line [8] is used in this section. In order to see the effect of the height of $h$ on the transmission characteristics between the two antennas, we change this distance from 1 to $10 \mathrm{~mm}$ and

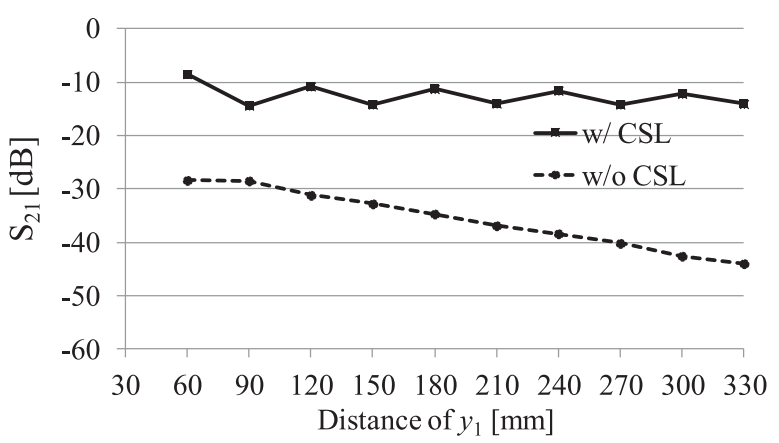

(a)

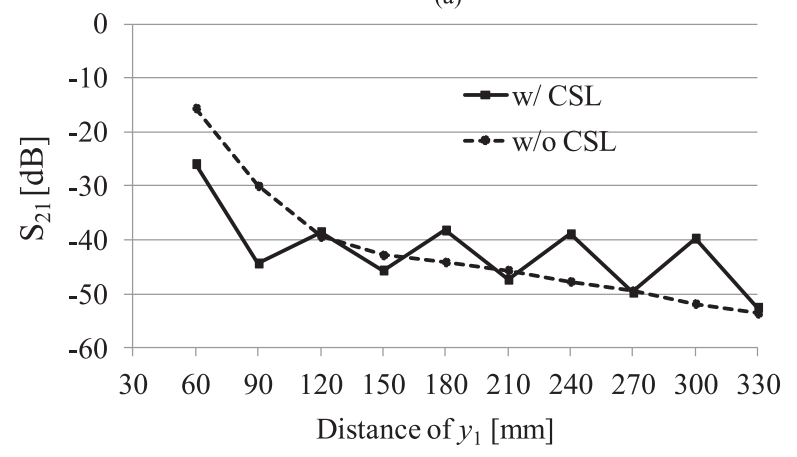

(b)

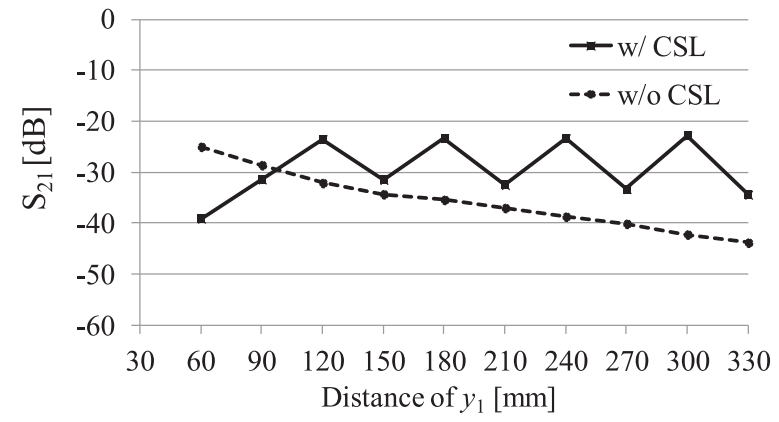

(c)

Fig. 3. Transmission characteristics between two patch antennas w/ CSL or w/o CSL having an equivalent dimension as the transmission line in [7] and [8] at $2.45 \mathrm{GHz}$. (a) Two antennas put below the CSL [see Fig. 2(a)]. (b) Two antennas placed above the CSL [see Fig. 2(b)]. (c) One antenna placed below, the other put above the CSL [see Fig. 2(c)].

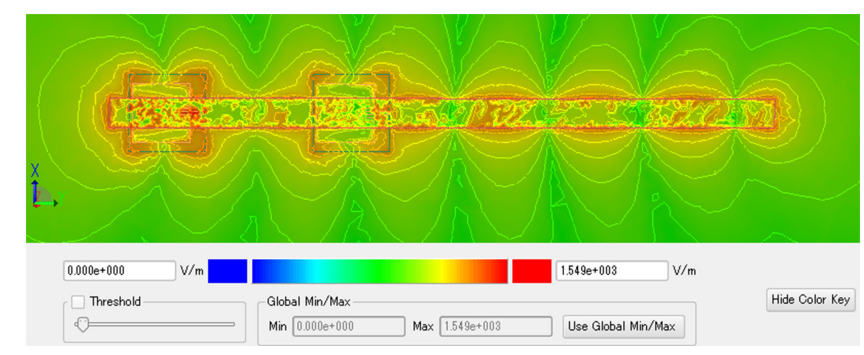

Fig. 4. Current surface on the silver-ink layer of the CSL at $y_{1}=90 \mathrm{~mm}$.

calculate $S_{21}$ when the antennas with the port 2 are moved along the CSL. Simulation results are shown in Fig. 6. The results on the PEC plane show that when $h$ increases, $\mathrm{S}_{21}$ decreases. But, the opposite trend can be seen with the results on the phantom model. The optimal value of $h$ on the PEC plane is $1 \mathrm{~mm}$, whereas it should be greater than $5 \mathrm{~mm}$ on the phantom model. However, the height of $h$ should be equal or greater 


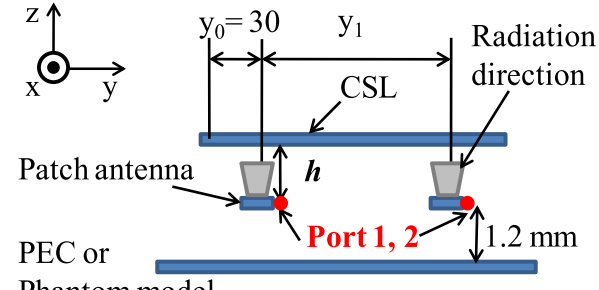

Phantom model

Fig. 5. Model utilized for simulation.

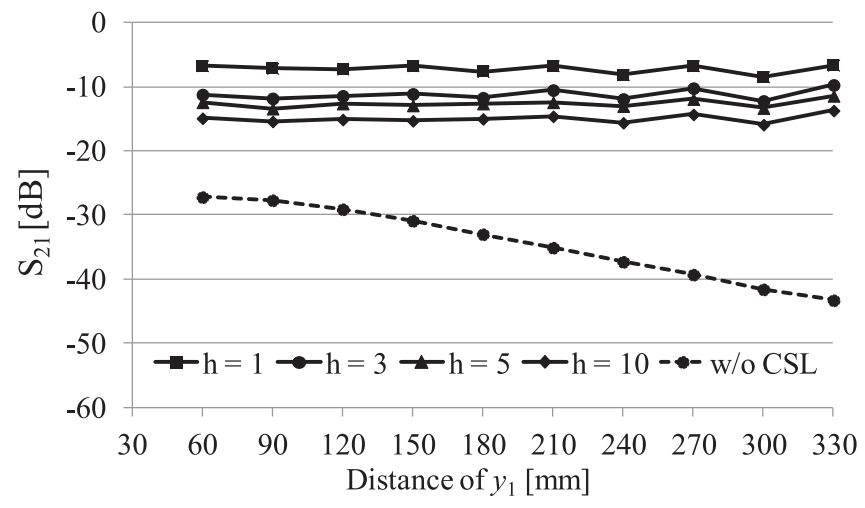

(a)

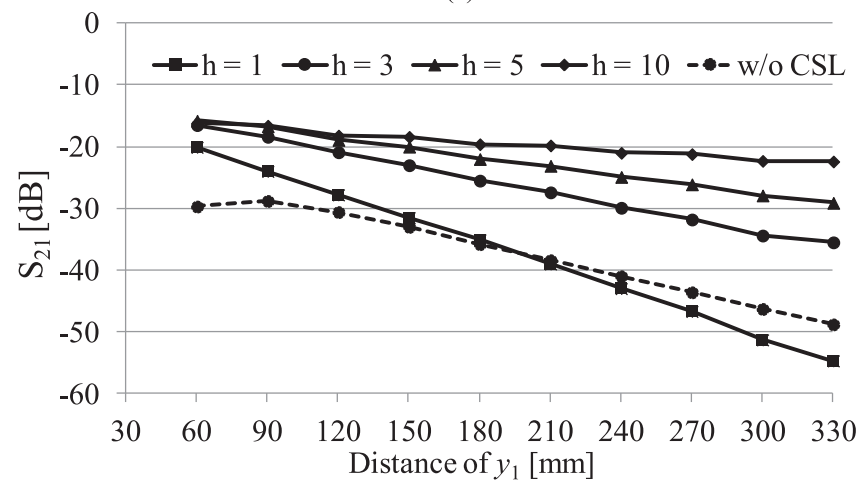

(b)

Fig. 6. Effect of the height of the CSL on the transmission characteristics between the two patch antennas at $2.45 \mathrm{GHz}$. (a) On the PEC plane. (b) On the phantom model.

than $5 \mathrm{~mm}$, considering practical applications, when the CSL is integrated into clothes.

\section{B. Optimal Width of $W$}

The optimal width of the CSL is considered in this section. Similarly, the distance between the antennas and the PEC plane is fixed at $1.2 \mathrm{~mm}$. We only change the width of the CSL from $6\left(0.05 \lambda_{0}\right)$ to $60 \mathrm{~mm}\left(0.5 \lambda_{0}\right)$. The optimal height of $h=5 \mathrm{~mm}$ in Section III-A is applied for this consideration. Simulation results are illustrated in Fig. 7. It can be seen in Fig. 7(a) that the effect of the width of the CSL on $\mathrm{S}_{21}$ is not significant. When the width decreases, $\mathrm{S}_{21}$ becomes more stable. Especially, when $W=6 \mathrm{~mm}$, the deviation in $\mathrm{S}_{21}$ is approximately zero. It is also true with simulation results on the phantom model. The results in Fig. 7(b) show that when the width increases, the performance of the CSL is reduced slightly. Thus, the optimal width of the CSL is $6 \mathrm{~mm}$ to obtain the highest performance, the stability of $\mathrm{S}_{21}$, and the high flexibility for on-body communication.

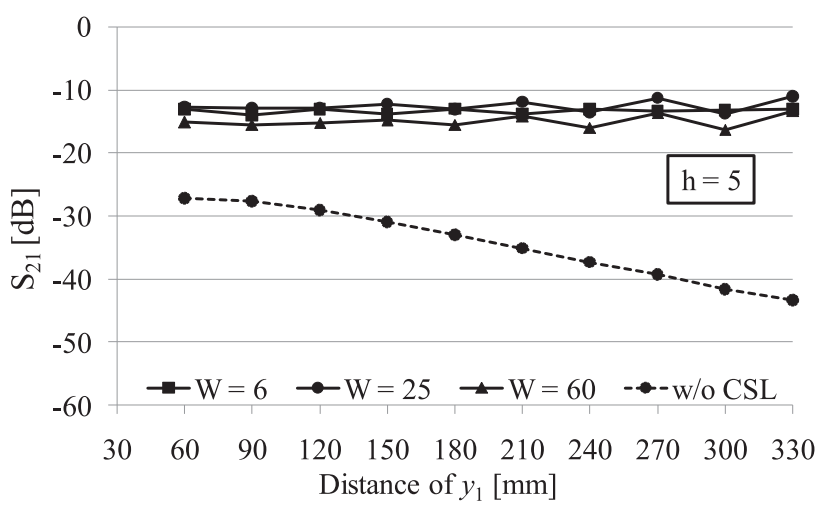

(a)

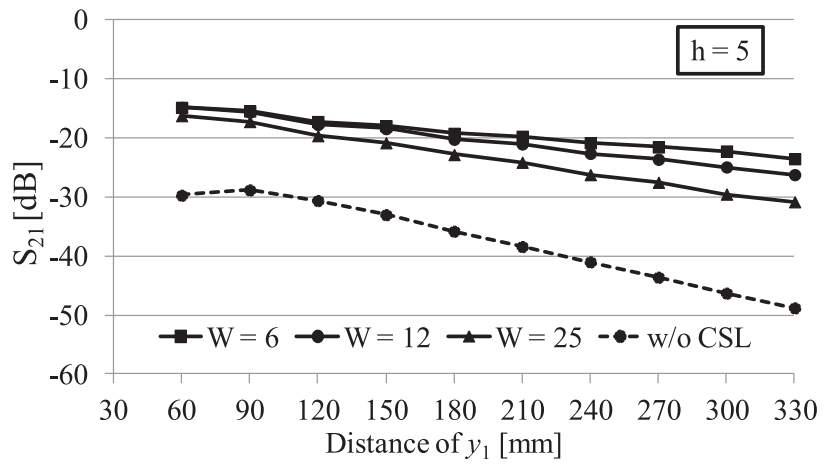

(b)

Fig. 7. Effect of the width of the CSL on the transmission characteristics between the two patch antennas at $2.45 \mathrm{GHz}$. (a) On the PEC plane. (b) On the phantom model.

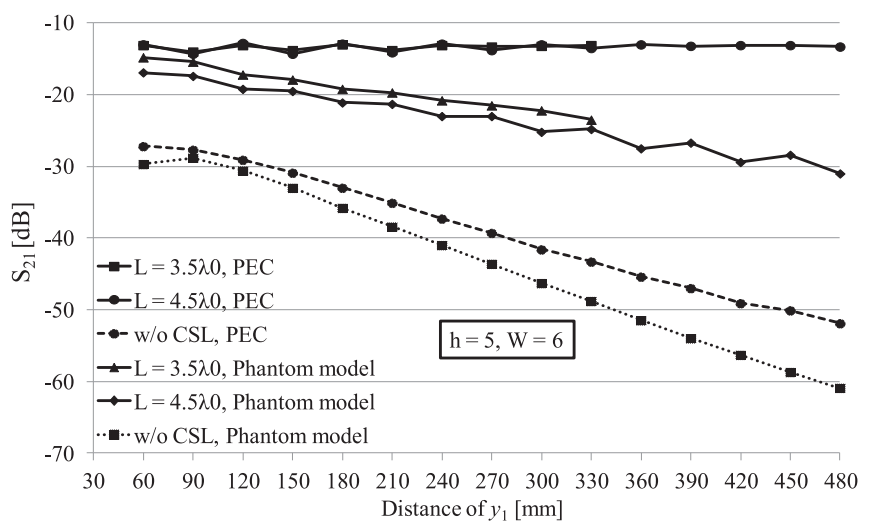

Fig. 8. Effect of the length of the CSL on the transmission characteristics between the two patch antennas at $2.45 \mathrm{GHz}$.

\section{Length of the CSL}

The performance of the longer CSL is simulated in this section. The length of the CSL is increased one wavelength from $3.5 \lambda_{0}$ to $4.5 \lambda_{0}$, which is long enough for practical on-body links. Simulation results are shown in Fig. 8. A higher improvement can be achieved when the distance of $y_{1}$ is increased.

\section{MEAsured Results}

The performance of the CSL is confirmed by measurements in this section. First, the measurement was done on the PEC plane. Then, we tested on a phantom and a real body. The CSL with the optimal parameters of $h=5$ and $W=6 \mathrm{~mm}$ and the length of $3.5 \lambda_{0}$ is used for these measurements. 


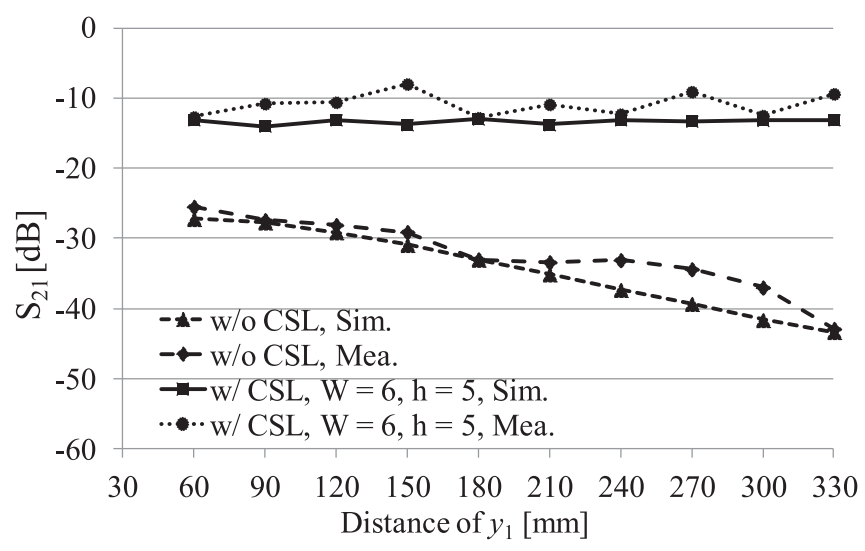

Fig. 9. Measured results of the transmission characteristics between two antennas on the PEC plane.

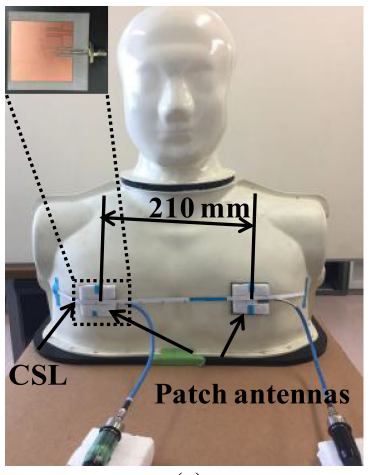

(a)

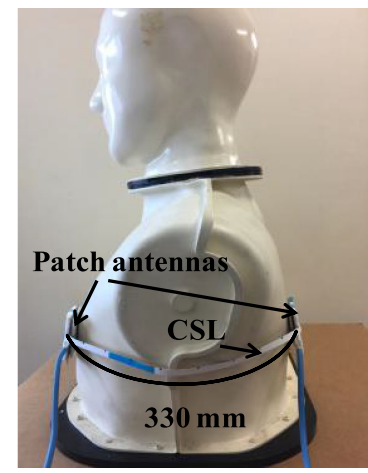

(b)
Fig. 10. Photographs of measurements on a phantom. (a) LOS case. (b) NLOS case.

\section{A. Measurement on the PEC Plane}

The measurement on the PEC plane was carried out in two cases: w/CSL and w/o CSL when $h=1 \mathrm{~mm}$. The measured results, as shown in Fig. 9, agree well with the simulation results.

\section{B. Measurement on a Phantom and a Real Body}

The transmission improvement between the two patch antennas by the CSL at $2.45 \mathrm{GHz}$ was demonstrated on an ultralight carbon upper body phantom [11] with the size of $280 \times 490$ $\times 220 \mathrm{~mm}^{3}$. The pictures of the propagation loss measurement between the two patch antennas are demonstrated in Fig. 10.

Two cases were measured: line-of-sight (LOS) case and nonline-of-sight (NLOS) case. In the LOS case, the two patch antennas were in front of the phantom with the distance of $210 \mathrm{~mm}$. In the NLOS case, one antenna was putted on the front side and the other was set on the back. The measured data are given in Fig. 11 and Table II. In a similar way, measurements on a real body were carried out and the results are also given in Fig. 11 and Table II. Through the measured results, the improvement by the CSL is significant, especially, for LOS case. Moreover, we can observe that the results on the phantom agree well with the results on the real body. Thus, the benefit of utilizing the CSL and its effectiveness for on-body communication was proved.

\section{SPECIFIC AbSORPtion RATE (SAR)}

In this section, we validate the SAR, which is an important requirement of any on-body devices for human safety. SAR is

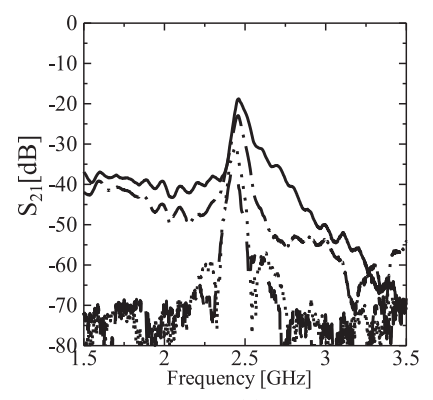

(a)

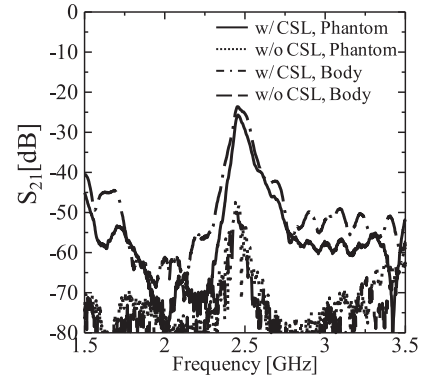

(b)
Fig. 11. Transmission losses between the two patch antennas on the phantom/the real body w/CSL and w/o CSL at $2.45 \mathrm{GHz}$ (real body: weight of $55 \mathrm{~kg}$, height of $1.7 \mathrm{~mm}$ ). (a) LOS case. (b) NLOS case.

TABLE II

TRANSMISSION GAIN BY THE CSL AT $2.45 \mathrm{GHz}$

\begin{tabular}{|c|c|c|c|c|c|}
\hline \multicolumn{6}{|c|}{ LOS case } \\
\hline \multicolumn{2}{|c|}{ Types of waveguide } & \multicolumn{2}{|c|}{ CSL } & \multicolumn{2}{|c|}{ SCPW [8] } \\
\hline \multicolumn{2}{|c|}{ Phantom/ Real body } & Phantom & $\begin{array}{l}\text { Real } \\
\text { Body }\end{array}$ & Phantom & $\begin{array}{l}\text { Real } \\
\text { Body }\end{array}$ \\
\hline \multirow{2}{*}{$\mathbf{S}_{21}$} & $\mathrm{w}$ & -19.5 & -23.3 & -22 & -41.4 \\
\hline & $\mathrm{w} / \mathrm{o}$ & -31.3 & -36.9 & -37.1 & -61.7 \\
\hline \multicolumn{2}{|c|}{ Transmission gain $[\mathrm{dB}]$} & 11.8 & 13.6 & 15 & 20.3 \\
\hline \multicolumn{6}{|c|}{ NLOS case } \\
\hline \multicolumn{2}{|c|}{ Types of waveguide } & \multicolumn{2}{|c|}{ CSL } & \multicolumn{2}{|c|}{ SCPW [8] } \\
\hline \multicolumn{2}{|c|}{ Phantom/ Real body } & Phantom & $\begin{array}{l}\text { Real } \\
\text { Body }\end{array}$ & Phantom & $\begin{array}{l}\text { Real } \\
\text { Body }\end{array}$ \\
\hline \multirow{2}{*}{$\mathbf{S}_{21}$} & $\mathrm{~W}$ & -26.3 & -23.8 & -25.7 & -29.8 \\
\hline & $\mathrm{w} / \mathrm{o}$ & -49.2 & -49.4 & -56.2 & -49.9 \\
\hline \multicolumn{2}{|c|}{ Transmission gain [dB] } & 22.9 & 25.6 & 29.5 & 20.1 \\
\hline
\end{tabular}

proportional to the power of the internal electric field $\left(|E|^{2}\right)$ and is expressed by

$$
\mathrm{SAR}=\frac{\sigma|E|^{2}}{\rho}
$$

where $\sigma$ and $\rho$ represent the electric conductivity $(\mathrm{S} / \mathrm{m})$ and mass density $\left(\mathrm{kg} / \mathrm{m}^{3}\right)$ of the medium, respectively [14].

However, for simplification, the simplified phantom model (mentioned in Section III) is used to get the maximum amplitudes of electric field $\left(E_{\max }\right)$ on its surface at points of $y_{1}$. Besides, the average mass density of human body $\rho=985 \mathrm{~kg} / \mathrm{m}^{3}$ [13] is applied. Thus, (1) is simplified as follows:

$$
\mathrm{SAR}=\frac{1.8\left|E_{\max }\right|^{2}}{985}
$$

Calculated results show that when the transmitting powers of the two patch antennas are -9.5 and $-8.4 \mathrm{dBm}$, acceptable powers for on-body communication [12], [15], the SAR is satisfied current standards of Europe and Japan [14], respectively.

\section{CONCLUSION}

The performance of the CSL in enhancing on-body communication was confirmed. Because of an extremely simple structure, high transmission improvement, and satisfied SAR requirement, the CSL is really effective for on-body links; especially for the NLOS links, the transmission gain is more than $20 \mathrm{~dB}$. 


\section{REFERENCES}

[1] P. S. Hall et al., "Antennas and propagation for on-body communication system," IEEE Antennas Propag. Mag., vol. 49, no. 3, pp. 41-58, Jun. 2007.

[2] A. Pellegrini et al., "Antennas and propagation for body-centric wireless communications at millimeter-wave frequencies: A review," IEEE Antennas Propag. Mag., vol. 55, no. 4, pp. 262-287, Aug. 2013.

[3] C. Mikeka and H. Arai, "Novel wearable sensors for body area network applications," in Microwave and Millimeter Wave Circuits and Systems: Emerging Design, Technologies, and Applications, 1st ed. Hoboken, NJ, USA: Wiley, 2012.

[4] H. Arai, "Free access transmission line for body centric communication (Invited)," in Proc. IEEE MTT-S Int. Microw. Workshop Ser. RF Wireless Technol. Biomed. Healthcare Appl., Taipei, Taiwan, 2015, pp. 136-137.

[5] Y. Shinozaki, T. Okura, and H. Arai, "Coupling characteristics between two dipole antennas over free access transmission line using paper substrate," in Proc. Int. Symp. Antennas Propag., Okinawa, Japan, 2016, pp. 310-311.

[6] Y. Shinozaki and H. Arai, "Free access transmission line using paper substrate in body area network," in Proc. Int. Workshop Electromagn., Appl. Student Innov. Competition, 2017, pp. 176-177.

[7] T. L. Tran, Y. Shinozaki, and H. Arai, "A flexible transmission line using coplannar waveguide for on-body links," in Proc. Int. Symp. Antennas Propag., Phuket, Thailand, 2017.

[8] T. L. Tran, Y. Shinozaki, T. Okura, and H. Arai, "A free-access segmented coplanar waveguide for on-body communication," IEEE Trans. Antennas Propag., vol. 66, no. 9, pp. 4524-4532, Sep. 2018, doi: 10.1109/TAP.2018.2842302.
[9] EMPro Software. 2018. [Online]. Available: http://www.keysight.com/en/ pc-/empro-3d-em-simulation-software $? \mathrm{cc}=\mathrm{GB} \& \mathrm{lc}=\mathrm{eng}$

[10] K. Fukunaga, S. Watanabe, Y. Yamanaka, H. Asou, Y. Ishii, and K. Sato, "Dielectric properties of liquid phantoms for evaluations of mobile phones," in Proc. IEICE EMC, Sendai, Japan, 2004, pp. 805-808.

[11] Phantom. 2018. [Online]. Available: http://www.mwf.co.jp

[12] S. Xiao, V. Sivaraman, and A. Burdett, "Adapting radio transmit power in wireless body area sensor networks," in Proc. ICST 3rd Int. Conf. Body Area Netw., 2008, Art. no. 14.

[13] W. Bauer and G. D. Westfall, University Physics With Modern Physics. New York, NY, USA: McGraw-Hill, 2011, p. 451.

[14] D. Thanh Le, L. Hamada and S. Watanabe, "A fast estimation technique for evaluating the specific absorption rate of multiple-antenna transmitting devices," IEEE Trans. Antennas Ptopag., vol. 65, no. 9, pp. 1947-1957, Apr. 2017.

[15] Mote Processor Radio \& Mote Interface Boards User Manual. [Online]. Available:https://www.cs.wmich.edu/gupta/teaching/cs5950/fall2011/mi ca\%20pinouts\%20from\%20memsic\%20mpr-mib_series_users_manual\% 207430-0021-09_a-t.pdf

[16] C. A. Balanis, "Microstrip antennas," in Antenna Theory, 3rd ed. Hoboken, NJ, USA: Wiley, 2005, pp. 816-843.

[17] Y. Yao, J. Zheng, and Z. Feng, "Diversity measurements for on-body channels using a tri-polarization antenna at $2.45 \mathrm{GHz}, "$ IEEE Antennas Wireless Propag. Lett., vol. 11, pp. 1285-1288, 2012.

[18] P. B. Samal, P. J. Soh, and G. A. E. Vandenbosch, "UWB all-textile antenna with full ground plane for off-body WBAN communications," IEEE Trans. Antennas Propag., vol. 62, no. 1, pp. 102-108, Jan. 2014. 